\title{
INSUFICIÊNCIA RENAL CRÔNICA: ANÁLISE QUALITATIVA SOBRE SAÚDE BUCAL
}

\section{Cléa Adas Saliba Garbin}

Docente Titular do Programa de Pós-Graduação em Odontologia Preventiva e Social, Universidade Estadual Paulista (UNESP), Araçatuba (SP), Brasil.

\section{Marcelo Augusto Amaral}

Doutorando do Programa de Pós-Graduação em Odontologia Preventiva e Social, Universidade Estadual Paulista (UNESP), Araçatuba (SP), Brasil.

\section{Aline Maria Malachini Miotto}

Docente Adjunta do Programa de Pós-Graduação em Ciência da Computação, Universidade Estadual de Maringá (UEM), Maringá (PR), Brasil.

\section{Artênio José Ísper Garbin}

Docente Adjunto do Programa de Pós-Graduação em Odontologia Preventiva e Social, Universidade Estadual Paulista (UNESP), Araçatuba (SP), Brasil

\section{Tania Adas Saliba}

Docente Adjunto do Programa de Pós-Graduação em Odontologia Preventiva e Social, Universidade Estadual Paulista (UNESP), Araçatuba (SP), Brasil
Autor correspondente

Cléa Adas Saliba Garbin cgarbin@foa.unesp.br
RESUMO: Objetivou-se avaliar a autopercepção de doentes renais crônicos sobre saúde bucal e analisar os fatores envolvidos no tratamento da insuficiência renal. Trata-se de uma pesquisa qualitativa, com o emprego da técnica de grupo focal. Foram realizados dois grupos focais com uma amostra de 12 doentes renais crônicos, com a participação de pesquisador/moderador, observador e seis participantes em cada grupo e um roteiro com questões norteadoras. Os discursos foram gravados e transcritos, os conteúdos textuais foram processados no software IRAMUTEQ e analisados pela técnica de classificação hierárquica descendente (CHD) e análise de conteúdo. Seis categorias emergiram da análise multivariada pela CHD: (1) Sofrimento; (2) Expectativa; (3) Acesso ao Tratamento; (4) Serviços de Saúde; (5) Valorização; (6) Funções da Boca. A análise qualitativa demonstrou que apesar do grande sofrimento relatado pelos doentes renais crônicos, há uma autopercepção positiva e que pode ser verificada nas categorias elencadas. A análise interpretativa do corpus textual sugere ausência de assistência odontológica e alguns fatores envolvidos neste processo de iniquidade.

PALAVRAS-CHAVE: Análise qualitativa; Insuficiência renal crônica; Saúde bucal.

\section{CHRONIC KIDNEY INSUFFICIENCY: QUALITATIVE ANALYSIS ON MOUTH HEALTH}

ABSTRACT: Self-perception by chronic kidney patients on mouth health is evaluated and the factors involved in the treatment of kidney insufficiency are analyzed. Qualitative research with focal group technique was applied to two groups with a sample of 12 chronic kidney patients, with the participation of researcher/moderator, observer and six participants of each group and a list of questions. Discourses were recorded and transcribed, textual contents were processed by IRAMUTEQ and analyzed by descendent hierarchized classification technique (CHD) and content analysis. Six categories emerged from the multivariate CHD analysis: (1) suffering; (2) expectations; (3) access to treatment; (4) health services; (5) valorization; (6) mouth functions. Qualitative analysis revealed that, in spite of great suffering by chronic kidney patients, a positive self-perception is perceived and may be verified in the listed categories. The interpretative analysis of the textual corpus suggests an absence of dentistry assistance and other factors involved in the iniquity process.

KEY WORDS: Qualitative analysis; Chronic kidney insufficiency; Mouth health. 


\section{INTRODUÇÃO}

A insuficiência renal crônica (IRC) é uma doença sistêmica, relativamente comum, que implica na redução ou limitação da capacidade de filtração glomerular dos rins, causando a uremia, que se caracteriza pelo acúmulo no sangue de substâncias que deveriam ser filtradas e excretadas pelos rins, ocorrendo o comprometimento deste órgão e levando o paciente a fazer uso da hemodiálise ${ }^{1}$. A prevalência e incidência de pacientes em diálise no Brasil tem aumentado entre os anos 2011-2014².

A IRC pode ocasionar determinadas mudanças sistêmicas, como problemas cardiovasculares (hipertensão arterial, aterosclerose, pericardites, cardiomiopatias, arritmias cardíacas e hipertensão pulmonar), anemia, problemas hemostáticos e linfocitopenia ${ }^{3}$, bem como pode ser consequência destas alterações.

Além das manifestações sistêmicas que acometem o paciente com IRC, as mudanças metabólicas e fisiopatológicas associadas à doença e as diferentes modalidades de tratamento podem afetar a cavidade bucal ${ }^{4}$. Têm sido relatados na literatura inflamação e aumento gengival $^{5}$, xerostomia, candidíase e mudanças no paladar ${ }^{6} \mathrm{e}$ doenças periodontais 7 .

Também são relatadas deficiência nos hábitos de higiene que podem afetar a saúde bucal destes pacientes, resultando em cáries e lesões bucais ${ }^{8}$, além de baixo fluxo salivar e elevação do pH da saliva ${ }^{9}$.

A presença de cáries, lesões endodônticas, abscessos, periodontite, pericoronarite, mucosites e peri-implantites servem como porta de entrada de microrganismos para a corrente sanguínea, podendo levar a um aumento da morbidade e potencial de mortalidade de pacientes com IRC submetidos à hemodiálise ${ }^{10}$. Indivíduos sistemicamente saudáveis podem ser mais facilmente controlados, no entanto, pacientes imunocomprometidos podem apresentar maior dificuldade para combater tais infecções ${ }^{11}$.

A maioria dos trabalhos na literatura focaliza os fatores de risco da IRC sobre a condição bucal dos doentes renais, mostrando apenas dados clínicos e epidemiológicos, muitas vezes sem avaliar o grau de conhecimento destes pacientes sobre a importância da saúde bucal ${ }^{12}$.

A pesquisa qualitativa em saúde tem potencial para dar respostas a problemas que não são solucionados com abordagens epidemiológicas, experimentais e outros desenhos de base quantitativa ${ }^{13}$. Ela oferece a possibilidade de produção de conhecimento aprofundado sobre fenômenos humanos, contribuindo para o entendimento da dimensão subjetiva presente no adoecimento e na dinâmica do processo saúde-doença, não traduzível em números. A investigação de natureza qualitativa se ocupa do nível subjetivo e relacional da realidade social, se apropriando das histórias, experiências, significados, motivos, crenças e valores dos atores sociais ${ }^{14}$.

A despeito da grande expansão verificada e dos aportes à saúde coletiva, a pesquisa qualitativa ainda se depara com vários desafios para a sua plena consolidação, com vistas a um modelo de ciência que responda à diversidade e complexidade que o caracterizam ${ }^{15}$.

Com base na necessidade de elaboração de estratégias eficazes que garantam a assistência adequada aos doentes renais, estudos que estabeleçam relações entre conhecimentos e percepções destes pacientes sobre 0 processo saúde-doença-tratamento são fundamentais. Nesta pesquisa objetivou-se avaliar a autopercepção de doentes renais crônicos sobre saúde bucal e analisar os fatores envolvidos no tratamento da insuficiência renal.

\section{METODOLOGIA}

\section{TIPO DE ESTUDO, COLETA E ENTREVISTAS}

Trata-se de uma pesquisa qualitativa com emprego da técnica de grupo focal. A amostra foi composta por 12 doentes renais crônicos. Foram incluídos na pesquisa doentes renais em período de diálise que concordaram em participar das entrevistas e apresentaram boa capacidade de comunicação.

A coleta de dados ocorreu no ano de 2017, nas dependências de um serviço especializado em doença renal crônica do município de Maringá (PR). Foram realizados dois grupos focais com seis pacientes cada, duração de 40 a 60 minutos, com a participação de um pesquisador/moderador e um observador.

No início da reunião com cada grupo focal, foram adotadas as seguintes instruções: apresentação do 
pesquisador/moderador e observador; explicações dos objetivos da pesquisa e da técnica usada; solicitação de permissão para uso do gravador ou similar; explicações da importância na organização das falas para as atividades do grupo, de forma que se evitasse a sobreposição das falas e, posteriormente, possíveis dificuldades na compreensão das gravações; ratificação sobre a importância da participação de todos do grupo; esclarecimento sobre o tempo de duração da reunião e confirmação de sua participação e distribuição de um número aos participantes $^{16}$, para que fossem preservadas as identidades e, assim, garantir o anonimato em cumprimento da Resolução 466/2012.

Para o desenvolvimento da técnica o pesquisador principal do estudo conduziu a entrevista orientando-se por um roteiro de tópicos, elaborado no sentido de nortear a conversa e apreender o ponto de vista dos sujeitos entrevistados sobre a temática "O olhar dos doentes renais crônicos sobre a saúde bucal”. Durante toda a entrevista, o pesquisador permitiu o livre discurso e ao mesmo tempo o delineamento da conversa, buscando manter a entrevista sintonizada com os objetivos da pesquisa. As falas da entrevista foram gravadas em aparelho digital e para seu encerramento utilizou-se o critério de saturação, isto é, quando, após as informações coletadas com certo número de participantes, novas entrevistas passaram a apresentar uma quantidade de repetições em seu conteúdo ${ }^{17}$.

Foram aplicadas cinco questões norteadoras para o grupo focal e vários complementos (Quadro 1). Encerradas as sessões, as gravações foram transcritas na íntegra, logo no dia seguinte da realização dos grupos focais.
Quadro 1. Tópicos e questões norteadoras do roteiro aplicado nos grupos focais. Maringá, Paraná (2017).

\begin{tabular}{|c|c|}
\hline Tópico & Questões \\
\hline \multirow{5}{*}{ Dor dentária } & 1) Você tem ou teve dores de origem dentária? \\
\hline & 1.1) Há quanto tempo? \\
\hline & 1.2) Onde você obteve tratamento? \\
\hline & 1.3) Por quanto tempo tem ou teve esta dor? \\
\hline & 1.4) A dor dentária interferiu na sua vida? \\
\hline \multirow{3}{*}{$\begin{array}{l}\text { Tratamento } \\
\text { odontológico }\end{array}$} & $\begin{array}{l}\text { 2) Você realiza atualmente algum tratamento } \\
\text { odontológico? }\end{array}$ \\
\hline & 2.1) Se não, por quê? \\
\hline & 2.2) Se sim, como foi? \\
\hline \multirow{3}{*}{ Saúde bucal } & 3) Você acha importante sua saúde bucal? \\
\hline & 3.1) E de seus familiares? \\
\hline & $\begin{array}{l}\text { 3.2) Como você acha que está a sua condição } \\
\text { bucal? }\end{array}$ \\
\hline \multirow{3}{*}{$\begin{array}{l}\text { Relação IRC X } \\
\text { Saúde bucal }\end{array}$} & $\begin{array}{l}\text { 4) Você acha que a doença renal tem relação } \\
\text { com a saúde bucal? }\end{array}$ \\
\hline & $\begin{array}{l}\text { 4.1) O tratamento de diálise interfere na sua } \\
\text { condição bucal? }\end{array}$ \\
\hline & $\begin{array}{l}\text { 4.2) E os medicamentos causam algum pro- } \\
\text { blema ou desconforto? }\end{array}$ \\
\hline \multirow{4}{*}{$\begin{array}{l}\text { Relação IRC X } \\
\text { Serviço odonto- } \\
\text { lógico }\end{array}$} & $\begin{array}{l}\text { 5) A sua condição atual interfere no acesso ao } \\
\text { serviço odontológico? }\end{array}$ \\
\hline & 5.1) Como seria um bom tratamento? \\
\hline & 5.2) Onde seria realizado este atendimento? \\
\hline & $\begin{array}{l}\text { 5.3) Já recebeu este tipo de tratamento em } \\
\text { algum local público ou particular? }\end{array}$ \\
\hline
\end{tabular}

\section{PROCESSAMENTO E ANÁLISE DOS DADOS}

Para o processamento dos dados, utilizou-se o software IRAMUTEQ (Interface de R pour les Analyses Multidimensionnelles de Textes et de Questionnaires) versão 0.7. Trata-se de um software livre que se ancora na linguagem de programação R, e que permite processamento e análises estatísticas de corpus textuais diversos ${ }^{18}$.

Para análise do conteúdo textual foi empregada a classificação hierárquica descendente (CHD), método proposto por Reinert ${ }^{19}$, que visa obter classes de palavras a partir de corpus textuais que, de forma concomitante, apresentam significados/vocabulários semelhantes entre si, mas, ao mesmo tempo, diferentes das outras classes. A CHD organiza a análise qualitativa dos textos em um dendograma, que apresenta graficamente as classes e suas possíveis relações. O pesquisador nomeia as classes de acordo com a semântica, considerando as frequências 
e os testes estatísticos Qui-quadrado $\left(\chi^{2}\right)$ fornecidos pelo software.

Após o processamento do conteúdo textual, construiu-se o modelo analítico composto por categorias, que corresponderam às classes de palavras geradas pelo IRAMUTEQ e CHD. As categorias podem ser estabelecidas antes do trabalho de campo, na fase exploratória da pesquisa, ou a partir da coleta de dados, e nesta pesquisa optou-se pela utilização destas categorias analíticas pós-coleta por serem mais específicas, concretas e por critério léxico ${ }^{14}$.

$\mathrm{Na}$ abordagem quantitativa, calcularam-se as frequências das palavras no conteúdo do texto, e na análise qualitativa foi considerado o conjunto de características em um determinado fragmento do conteúdo ${ }^{20}$. A análise interpretativa do corpus se deu pelo uso da análise de conteúdo, por poder ser esta quantitativa e qualitativa.

A análise qualitativa cumpriu as etapas: transcrição e digitação das gravações das entrevistas; atribuição de códigos aos entrevistados; leitura compreensiva dos textos; elaboração de estruturas de análise, agrupando trechos de depoimentos mais ilustrativos nos eixos temáticos; identificação das ideias centrais; identificação dos sentidos atribuídos às ideias; e elaboração de sínteses compreensivas, interpretativas e contextualizadas ${ }^{21}$.

\section{ASPECTOS ÉTICOS}

Todos os participantes assinaram o Termo de Consentimento Livre e Esclarecido e a pesquisa foi aprovada pelo Comitê de Ética em Pesquisa do Centro Universitário de Maringá (UniCesumar), segundo o parecer $\mathrm{n}^{\circ} 1.672 .905 / 2016$.

\section{RESULTADOS E DISCUSSÃO}

Quanto ao perfil dos doentes renais crônicos, três eram do gênero feminino e nove do gênero masculino. A média de idade dos doentes com IRC, no momento da realização da pesquisa, era de 56,58 $\pm 12,11$ anos, variando de 34 a 73 anos. O tempo de diálise foi, em média, de 44,75 \pm 32,01 meses, sendo encontrados pacientes de oito a 123 meses de tratamento. Dos 12 pais participantes, oito eram casados e brancos e somente um destes era portador de diploma de ensino superior (Tabela 1).
Tabela 1. Caracterização dos doentes renais crônicos, especificando gênero, idade, tempo de diálise, estado civil, cor da pele e escolaridade. Maringá, Paraná (2017).

\begin{tabular}{|c|c|c|c|c|c|c|}
\hline 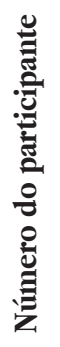 & 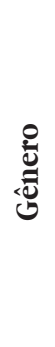 & 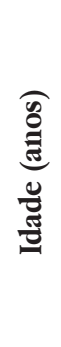 & 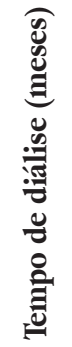 & 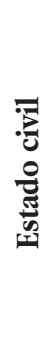 & 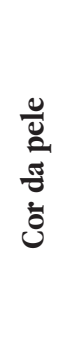 & 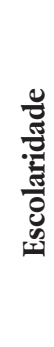 \\
\hline 1 & $\mathrm{M}$ & 72 & 77 & $\mathrm{C}$ & $\mathrm{N}$ & $\mathrm{FC}$ \\
\hline 2 & $\mathrm{M}$ & 58 & 54 & $\mathrm{C}$ & B & FI \\
\hline 3 & $\mathrm{M}$ & 67 & 31 & $\mathrm{C}$ & $\mathrm{P}$ & FI \\
\hline 4 & $\mathrm{~F}$ & 56 & 123 & $\mathrm{C}$ & B & A \\
\hline 5 & $\mathrm{M}$ & 38 & 56 & $\mathrm{~S}$ & B & MI \\
\hline 6 & $\mathrm{M}$ & 73 & 58 & $\mathrm{D}$ & $\mathrm{AM}$ & FI \\
\hline 7 & $\mathrm{M}$ & 60 & 42 & $S$ & B & MI \\
\hline 8 & $\mathrm{~F}$ & 34 & 28 & $\mathrm{C}$ & $\mathrm{P}$ & $\mathrm{MC}$ \\
\hline 9 & $\mathrm{M}$ & 47 & 14 & $\mathrm{C}$ & B & MC \\
\hline 10 & $\mathrm{~F}$ & 56 & 23 & $\mathrm{C}$ & B & $\mathrm{SC}$ \\
\hline 11 & M & 63 & 23 & $S$ & B & A \\
\hline 12 & $\mathrm{M}$ & 59 & 08 & $\mathrm{C}$ & B & $\mathrm{MC}$ \\
\hline
\end{tabular}

A: Analfabeto; AM: Amarelo; B: Branco; C: Casado; D: Divorciado; F: Feminino; FC: Fundamental completo; FI: Fundamental incompleto; M: Masculino; MC: Médio completo; MI: Médio incompleto; N: Negro: P: Pardo; S: Separado; SC: Superior completo.

\section{CARACTERIZAÇÃO DAS CLASSES/CATEGORIAS}

$\mathrm{Na}$ análise do corpus "O olhar dos doentes renais crônicos sobre saúde bucal”, proveniente da transcrição dos grupos focais, foram observadas 8.801 ocorrências de palavras, sendo 1.277 formas distintas, com frequência média de 3,26 palavras para cada forma. Esse corpus foi dividido em 256 unidades de contexto elementares e, destas, 213 , ou seja, $83,2 \%$ do total de palavras foram equiparadas por meio de classificações hierárquicas descendentes de segmentos de texto de tamanhos diferentes, indicando o grau de semelhança no vocabulário dos seis temas resultantes. Na Figura 1 pode-se visualizar o dendograma que demonstra as classes/categorias advindas das partições do conteúdo. 


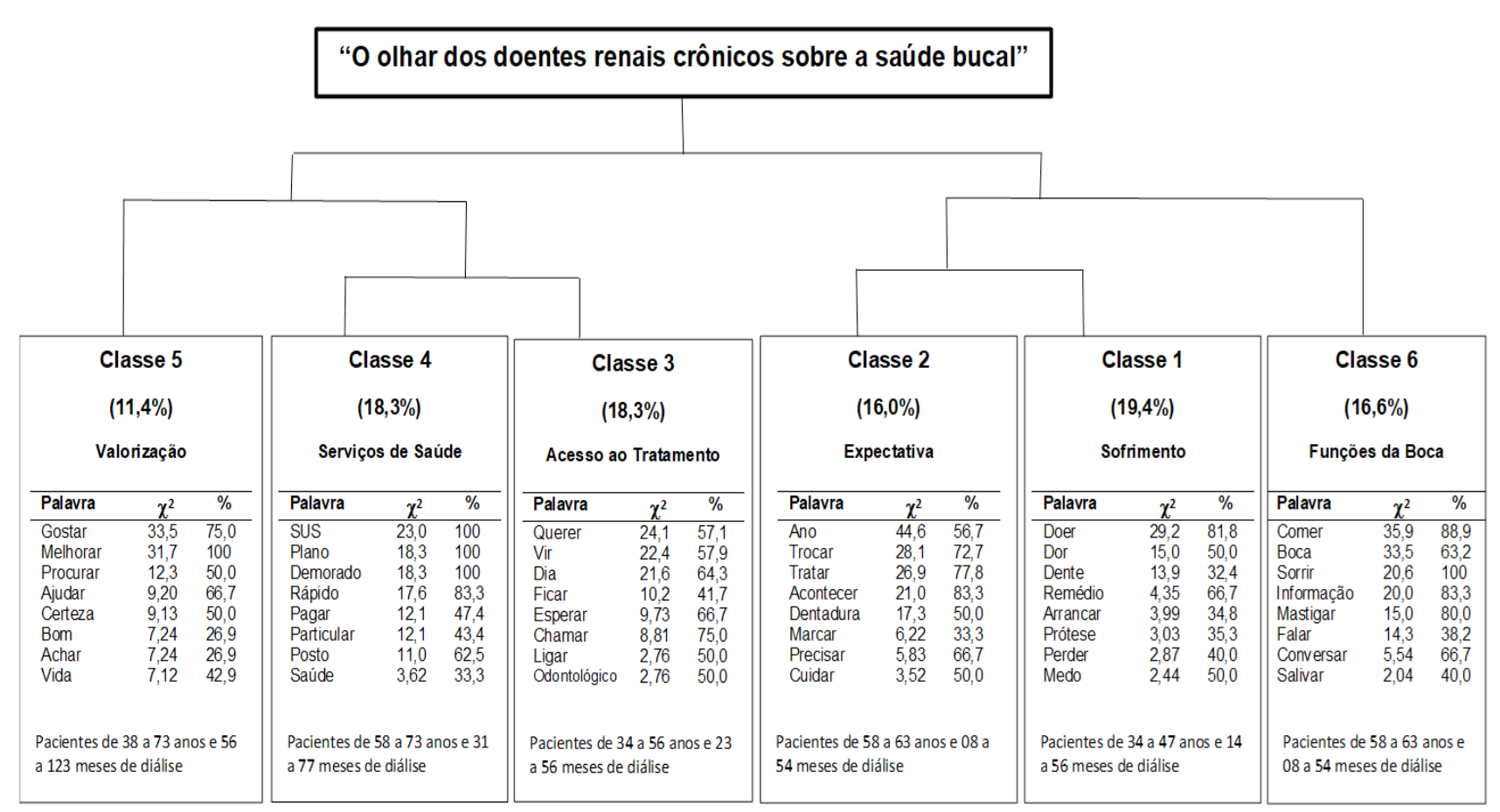

Figura 1. Dendograma da Classificação Hierárquica Descendente com as partições e conteúdo do corpus da pesquisa.

Observou-se convergência das características empíricas em torno de seis temas: a) sofrimento; b) expectativa; c) acesso ao tratamento; d) serviços de saúde; e) valorização; e f) funções da boca.

A seguir é apresentada uma análise interpretativa no contexto de cada uma das categorias identificadas e agrupadas a partir da aplicação da técnica CHD no corpus textual.

\section{ANÁLISE INTERPRETATIVA: O OLHAR DOS DOENTES RENAIS}

\section{SOFRIMENTO}

A categoria sofrimento foi a mais expressiva do grupo focal. As palavras mais representativas foram: doer, dor, dente, remédio, arrancar, prótese, perder e medo. Esta categoria evidencia possíveis fatores que poderiam levar a uma percepção negativa ou desinteresse pela procura do atendimento odontológico. Nesta categoria também há indicativos da associação entre uma experiência de tratamento emergencial (arrancar e perder) e o medo (por algum fator que gere ansiedade e/ou estresse neste procedimento clínico). Algumas falas dos doentes renais ajudam a compreender de forma ilustrativa o conteúdo da classe e o contexto de seus elementos:

[...] e o dente doeu, aí eu peguei fui na farmácia e comprei um remédio, tomei, mas estava inflamado e não fiz o tratamento do jeito certo e só passou a dor quando arranquei 0 dente (DRC 5).

[...] a dor de dente interferiu na minha vida porque tive que arrancar meus dentes, se não tivesse doendo não ia arrancar e quem me atendia era dentista formado. Depois eu comecei a fazer tratamento em outro local e sem medo (DRC 8).

[...] eu paguei para fazer um tratamento em outra cidade e lá não fazia prótese, até fazia, mas, era mais muito caro! Na época, não tinha muita dor de dente e agora tive que arrancar os dentes e tenho duas próteses na boca (DRC 9).

Deste modo, é de fundamental importância definir os motivos que levam ao abandono ou descontinuidade de um tratamento odontológico, com o intuito de refletir sobre novas práticas de saúde para os portadores de IRC, bem como outras populações de enfermos. 
Da mesma forma, Corrêa et al..$^{22}$ relatam que a presença de dor (na boca ou dentes) foi um fator diretamente associado à insatisfação com a condição de saúde bucal e autopercepção negativa dos usuários entrevistados.

Os indivíduos com necessidade de próteses dentárias tenderam a fazer uma avaliação negativa da sua saúde bucal. Observa-se que a necessidade de reabilitação protética é uma situação clínica que implica diretamente na qualidade da mastigação, bem como na autopercepção do sorriso, e gera, muitas vezes, constrangimento para falar e sorrir, além de dificuldades nas relações interpessoais ${ }^{23}$.

As representações sociais do cuidado à saúde bucal salientam as consequências negativas da prática odontológica vigente e apontam para a necessidade de reestruturação dos serviços, levando em conta a percepção da população em relação ao cuidado à saúde bucal24 .

\section{EXPECTATIVA}

As palavras mais representativas desta categoria foram: aguardar, trocar, tratar, acontecer, dentadura, marcar, precisar e cuidar. Esta classe evidenciou a situação de quem espera a ocorrência de algo, ou sua probabilidade de ocorrência, em determinado momento, que na perspectiva dos doentes renais se relaciona ao tratamento odontológico. A IRC provoca insegurança para estes pacientes diante de algo desconhecido e novo, inclusive sua condição bucal.

Os pacientes deste estudo apresentaram grande expectativa frente ao tratamento odontológico e esta necessidade e sentimentos de espera são indicados como fatores determinantes deste estado emocional, conforme relatos presentes nas falas ilustrativas a seguir:

[...] também preciso urgentemente de uma prótese total, porque a de cima eu tenho que trocar porque eu coloquei ela lá pelos anos noventa e dois e eu nunca troquei e estou disposto a procurar um dentista (DRC 2).

[...] antigamente ninguém quase cuidava dos dentes e ia ao dentista. Acho que a pessoa não cuidava dos dentes porque não tinha acesso aos dentistas e ao tratamento. Hoje quero tratar todos os meus dentes e tomara que consiga rápido (DRC 11). [...] já tive muita dor de dente no passado. Até que há muitos anos atrás eu comecei a tratar e o dentista levou muito tempo para acabar. Mas agora, tem uma parte do dente que foi arrancado e tem que colocar aparelho logo! E preciso cuidar da minha boca (DRC 12).

Diferentemente da expectativa ressaltada nas falas acima apresentadas, um estudo realizado com idosos em hemodiálise no interior do Estado de São Paulo, e em comparação com pacientes portadores de outras patologias crônicas, identificou um nível de esperança baixo, o que mostrou a necessidade da realização de intervenções reabilitadoras para que haja a melhora deste sentimento para esta população $0^{25}$.

Estudos sobre as expectativas e nível de esperança de doentes crônicos são raros, bem como o entendimento dos significados e aspirações destes pacientes. Nesse sentido, estudos que se propõem a conhecer as percepções de pacientes com relação à sua saúde bucal e necessidade de tratamento podem servir como referência para a elaboração de políticas de saúde para esses indivíduos $^{26}$.

\section{ACESSO AO TRATAMENTO}

A categoria acesso ao tratamento representa a segunda classe com maior expressão do grupo focal. Pode-se visualizar que as principais palavras dessa categoria foram: querer, vir, buscar, ficar, esperar, chamar, ligar e odontológico. Uma ilustração exemplifica o que os participantes revelam sobre o elemento "odontológico", e como estes resolvem suas necessidades de tratamento. Conforme pode ser observado no relato a seguir:

[...] o dentista do posto não quis arrancar o dente pra mim e ficou falando umas coisas lá e eu me chateei, vim embora e não voltei mais lá. Até que depois eu fui numa dentista particular e eu tive que pagar pra ela tirar o dente, porque não tinha outro jeito! (DRC 5). 
Segundo Almeida et al. ${ }^{26}$, a autopercepção da pessoa com doença crônica pode referir que a doença sistêmica não trouxe prejuízo para sua saúde bucal. Dessa forma, uma avaliação clínica deve servir como guia para a execução de políticas públicas que visem a favorecer um tratamento odontológico efetivo para esses pacientes e a execução de programas que promovam a saúde bucal deve ser estimulada, buscando-se desta maneira proporcionar uma atenção integral à saúde destas pessoas.

Nesse contexto, uma das falas apresentadas a seguir retrata as necessidades de tratamento não atendidas:

[...] porque a necessidade do ser humano e o corpo da gente precisa de cuidados integrais e isso é em primeiro lugar, porque não dá para esperar. Eu queria saber do dentista do postinho que dia eu vou começar o tratamento para a troca da prótese (dentadura) quebrada e antiga (DRC $10)$.

A falta de acesso à prótese dentária afeta o princípio da integralidade, e compromete também os princípios da universalidade e equidade. O cuidado em saúde deve abarcar suas diversas dimensões e deve oferecer respostas aos diversos grupos atingidos pelos problemas apresentados, o que não acontecerá sem a possibilidade do acesso à prótese dentária por meio do Sistema Único de Saúde ${ }^{27}$.

Independentemente do tipo de necessidade protética, é preciso avaliar como as pessoas percebem a funcionalidade e necessidade dessas próteses, e esta é uma característica determinante para a autopercepção da saúde bucal dos indivíduos ${ }^{22}$.

\section{SERVIÇOS DE SAÚDE}

A categoria serviços de saúde apresenta o mesmo percentual de representação da categoria anterior do grupo focal dos pacientes renais. As palavras que mais contribuíram foram: SUS, plano, demorado, rápido, pagar, particular, posto e saúde. No cotidiano destes doentes renais são evidenciadas muitas situações para diferentes análises do modelo assistencial vigente, como ilustram alguns participantes:
[...] para mim o sistema público sempre foi melhor que o sistema privado, para falar a verdade para você eu sempre fui atendido no postinho, e faço hemodiálise a quatro anos e meio! Antes eu pagava plano e não foi bom, então quero dizer que o SUS para mim foi muito bom (DRC 1).

[...] eu faço todas as coisas que preciso pelo SUS e para mim é ótimo! É como se fosse um plano de saúde para mim e minha família (DRC 2).

[...] o sistema público é diferente do plano, mais demorado e tem que agendar com muito tempo antes. No particular é mais rápido porque você pode fazer qualquer coisa sem ter que esperar às vezes um ano pra uma consulta de dentista (DRC 3).

[...] para minha esposa e meu filho eu pago plano de saúde, e eu não passo no plano de saúde devido a ser doente renal, então eu não acho $o$ atendimento deles melhor que o meu que às vezes não tem médicos bons (DRC 6).

Os movimentos de definição de um ou outro modelo de saúde caminham ora no sentido de conservação do modelo tradicional, ora no sentido de um novo modelo, ou ainda na convivência conflitiva ou complementar entre eles. A busca de um modelo assistencial que esteja orientado para as necessidades ampliadas de saúde, em sintonia com os princípios do SUS e que supere os problemas decorrentes da hegemonia do paradigma da biomedicina é um dos grandes desafios do sistema de saúde brasileiro na atualidade ${ }^{28}$.

No Brasil, houve diferentes modelos assistenciais, como: sanitarista campanhista; modelo assistencial privatista; e SUS; e que podem ser representados por algumas falas a seguir de doentes que vivenciaram tais experiências:

[...] antigamente não tinha nada de atendimento, tudo era muito difícil se um filho ou a gente ficava doente, não tinha médico, dentista nem hospital onde eu morava e só andando muito para encontrar ajuda (DRC 1).

[...] vivi na época do particular e

(n)


tudo só existia para quem pagava e tinha direito. Meu pai não tinha emprego com carteira e meus irmãos e eu passamos muitas dificuldades, inclusive perdendo vários parentes doentes que morreram (DCR 3).

[...] tudo que preciso tem no posto de saúde onde moro. Recebo até médico para cuidar da minha sogra que vive na cama e não consegue andar (DRC 6).

Assim, para construção de um serviço de saúde com potencial para romper com o paradigma do modelo atual, é importante considerar dois principais aspectos: o cotidiano das práticas assistenciais e as necessidades de saúde dos usuários ${ }^{28}$, como a dos doentes renais crônicos.

\section{VALORIZAÇÃO}

A categoria valorização foi a classe de menor expressão, originada na partição do corpus textual dos grupos focais. As palavras mais representativas a partir da utilização dos critérios de inclusão descritos anteriormente foram: gostar, melhorar, procurar, ajudar, certeza, bom, achar e vida. Contribuíram para esta categoria principalmente os doentes renais com maior tempo de diálise. Nesta classe pode-se verificar a autoavaliação dos doentes renais acerca dos sentimentos relacionados à saúde bucal, como exemplifica os trechos transcritos a seguir:

[...] eu acho que minha condição bucal está razoável, talvez tenha que fazer um tratamento dentário completo. E dá para melhorar e eu gostaria que tivesse dentista para nos atender e resolver aqueles probleminhas antigos (DRC 1).

[...] as propagandas na televisão falam que é bom tratar dos dentes para ficar com o sorriso bonito e tudo isso é qualidade de vida, principalmente para nós doentes dos rins e minha boca não está boa e gostaria muito de melhorar ela (DRC 4).

[...] é muito importante a saúde da boca e o dente da gente deveria ser em primeiro lugar, aí se você não consegue ter seus dentes tem que trocar ou ficar desdentado (DRC 5).
[...] eu gostaria muito de melhorar minha saúde da boca, e eu já fiz uma prótese inferior e não deu certo $\mathrm{e}$ não se adaptou muito bem e sei explicar. Queria muito ter condições de realizar um tratamento com tudo que preciso (DRC 6).

Verifica-se que os pacientes com insuficiência renal crônica apresentam uma percepção quanto à necessidade dos cuidados com a condição bucal. No entanto, estes pacientes não apresentam na equipe de saúde que os acompanha um profissional de Odontologia que os avalie regularmente e apresente um laudo odontológico de suas reais necessidades de tratamento, bem como realize o encaminhamento de acordo com suas especificidades bucais.

Em estudo realizado com doentes renais em hemodiálise, Valadares et al. ${ }^{12}$ relataram que apenas um em cada três dialíticos apresentavam um laudo odontológico com sua condição bucal. Deste modo, há necessidade de conscientizar as equipes de profissionais que atendem a pessoa com IRC e os pacientes renais sobre a importância de prevenir e/ou controlar as infecções bucais para a manutenção de sua saúde geral e realização e sucesso de um futuro transplante renal.

Segundo Portaria 389 do Ministério da Saúde ${ }^{29}$, a equipe mínima para atendimento em unidade especializada em doença renal crônica é composta por: médico nefrologista, enfermeiro, nutricionista, psicólogo e assistente social. Não se encontra incluído nesta equipe multiprofissional o cirurgião-dentista que pode realizar os laudos odontológicos, encaminhamentos e tratamentos de infecções bucais. Deve-se ressaltar que segundo os resultados do presente estudo tal inclusão é fundamental para a valorização da saúde bucal dos pacientes renais.

\section{FUNÇÕES DA BOCA}

As palavras mais representativas desta última categoria foram: comer, boca, sorrir, informação, mastigar, falar, conversar e salivar. Nesta classe pode-se comprovar as funções pré-percebidas da cavidade bucal pelos participantes deste estudo, bem como possibilitar uma reflexão da importância da boca, e que podem ser analisadas nas falas a seguir: 
[...] você vai num lugar, vai num evento ou qualquer lugar da cidade e se você não tem um dente bom você não pode comer uma carne, um churrasco! Você não pode comer nada de coisa boa (DRC 2).

[...] dente serve para tudo, para mastigar. Se a gente quer comer uma carne, para falar, conservando os dentes da boca você consegue conversar com qualquer um. Não ter mau cheiro na boca e já recebi alguma informação sobre saúde bucal (DRC 7).

[...] tem hora que tenho até inveja dos outros porque eu não posso sorrir, não posso comer e a fala também fica ruim! Uma coisa que eu gostava bastante era assoviar, mas eu não posso mais fazer isso infelizmente (DRC 11).

[...] a saúde da boca vem em primeiro lugar eu admiro quem tem tratamento dos dentes e consegue conversar normalmente (DRC 12).

A doença renal crônica continua sendo um problema de saúde pública prevalente que afeta desproporcionalmente as minorias e os indivíduos de menores condições econômicas, apesar dos esforços intensos direcionados ao controle dos fatores de risco tradicionais ${ }^{7}$. E pelas falas acima apresentadas, considera-se a importância do acesso ao atendimento odontológico para suprir as necessidades socais levantadas nesta pesquisa, que vão além do fator biológico do elemento dentário.

A importância dos cuidados com a saúde bucal no manejo de pacientes com doenças sistêmicas, incluindo a doença renal crônica, tem sido relatada. Muitos pacientes renais crônicos têm diferentes infecções bucais, no entanto, a atenção aos cuidados desta área tem faltado, especialmente nos países em desenvolvimento e com grande aumento na incidência desta doença ${ }^{6}$.

A complexidade dos cuidados para os doentes renais crônicos reforça a necessidade de colaboração entre os diversos profissionais pertencentes à equipe especializada. Além disso, a incorporação da educação interdisciplinar aumenta o conhecimento e troca de experiências entre profissionais e pode levar a melhores resultados na qualidade de vida e, consequentemente, melhorar a saú- de geral destes pacientes ${ }^{30}$, inclusive para os transplantados renais após longo período do transplante ${ }^{31}$.

\section{CONCLUSÕES}

As percepções dos doentes renais crônicos em relação à saúde bucal demonstram uma presente influência do sofrimento relatado nas mais diversas falas dos participantes dos grupos focais. Percebe-se uma autoavaliação positiva e de grande importância da condição bucal na vida destes pacientes. Entretanto, constatou-se dificuldades de acesso dos doentes renais a serviços de saúde com equipes multiprofissionais e iniquidades a assistência odontológica.

\section{REFERÊNCIAS}

1. Dias CRS, Sá TCV, Pereira ALA, Alves CMC. Avaliação da condição bucal em pacientes renais crônicos submetidos à hemodiálise. Rev Assoc Med Bras 2007;53(6):510-14.

2. Sesso RC, Lopes AA, Thomé FS, Lugon JR, Martins CT. Inquérito brasileiro de diálise crônica 2014. J Bras Nefrol 2016;38(1):54-61.

3. Stein A, Wild J. Kidney failure explained. $2^{\mathrm{a}}$ ed. London: Class Publishing; 2002.

4. Proctor R, Kumar N, Stein A, Moles D, Porter S. Oral and dental aspects of chronic renal failure. J Dent Res 2005;84(3):199-208.

5. Davidovich E, Schwarz Z, Davidovich M, Eildelman E, Bimstein E. Oral findings and periodontal status in children, adolescents and young adults suffering from renal failure. J Clin Periodontol 2005; 32(1):76-82.

6. Oyetola EO, Owotade FJ, Agbelusi GA, Fatusi OA, Sanusi AA. Oral findings in chronic kidney disease: implications for management in developing countries. BMC Oral Health 2015;15:24.

7. Grubbs V, Garcia F, Jue BL, Vittinghoff E, Ryder M, Lovett $\mathrm{D}$, et al. The kidney and periodontal disease 
(KAPD) study: a pilot randomized controlled trial testing the effect of non-surgical periodontal therapy on chronic kidney disease. Contemp Clin Trials 2017;53:143-50.

8. Barros DCP, Cordova LHS, Velandia ALP, Souza DM. Avaliação dos hábitos de higiene bucal de pacientes em hemodiálise do hospital regional do Vale do Paraíba. Braz J Periodontol 2014;24(3):7-11.

9. Lacerda MCSR, Viana KB, Dores DF, Ribeiro CMB, Bessa-Nogueira RV. Caracterização da saúde bucal de indivíduos renais crônicos aptos a transplante. Rev Odontol UNESP 2015;44(5):292-98.

10. Brunetti MC. Periodontia médica: uma abordagem integrada. São Paulo: Senac; 2004.

11. Nisengard RJ, Newman MG. Microbiologia oral e imunologia. Rio de Janeiro: Guanabara Koogan; 1997.

12. Valadares DM, Oliveira IRM, Parente RM, Cavaleiro RMS. The opinion of patients with chronic kidney disease about the importance of oral health - field research. Full Dent Sci 2013;4(15):465-71.

13. Green J, Thorogood N. Qualitative methods for health. $3^{\mathrm{a}}$ ed. London: SAGE; 2014.

14. Minayo MCS. O desafio do conhecimento: pesquisa qualitativa em saúde. $13^{\mathrm{a}}$ ed. Rio de Janeiro: Hucitec, Abrasco; 2013.

15. Bosi MLM. Pesquisa qualitativa em saúde coletiva: panorama e desafios. Cienc Saude Colet 2012; 17(3):575-86.

16. Moimaz SAS, Amaral MA, Miotto AMM, Costa ICC, Garbin CAS. Análise qualitativa do aleitamento materno com o uso do software IRAMUTEQ. Saúde e Pesquisa 2016;9(3):567-77.

17. Turato ER. Tratado de metodologia da pesquisa clínico-qualitativa: construção teórico-epistemológica, discussão comparada e aplicação nas áreas de saúde e humanas. $2^{\mathrm{a}}$ ed Petrópolis: Editora Vozes; 2003.

18. Ratinaud P. IRAMUTEQ: Interface de R pour les Analyses Multidimensionnelles de Textes et de Questionnaires [Computer Software]. 2009. [acessa- do 2018 mai 15]. Disponível em: <www.iramuteq. org $>$.

19. Reinert M. Alceste une méthodologie d'analyse des donnés textuelles et une application: Aurelia de Gerard de Nerval. Bull Methodol Sociol 1990;26: $24-54$.

20. Bardin L. Análise de Conteúdo. São Paulo: Edições 70; 2011.

21. Minayo MCS, Ribeiro AP. Condições de saúde dos presos do estado do Rio de Janeiro, Brasil. Cienc Saude Colet 2016;21(7):2031-40.

22. Corrêa HW, Bitencourt FV, Nogueira AV, Toassi RFC. Saúde bucal em usuários da atenção primária: análise qualitativa da autopercepção relacionada ao uso e necessidade de prótese dentária. Physis 2016;26(2):503-24.

23. Vale EB, Mendes ACG, Moreira RS. Autopercepção da saúde bucal entre adultos na região Nordeste do Brasil. Rev Saude Publica 2013:47(3):98-108.

24. Ferreira AAA, Piuvezam G, Werner CWA, Alves MSCF. A dor e a perda dentária: representações sociais do cuidado à saúde bucal. Cienc Saude Colet 2006;11(1):211-18.

25. Orlandi FS, Pepino BG, Pavarini SCI, Santos DA, Mediondo MSZ. Avaliação do nível de esperança de vida de idosos renais crônicos em hemodiálise. Rev Esc Enferm. USP 2012;46(4):900-5.

26. Almeida JRS, Alencar CH, Barbosa JC, Dias AA, Almeida MEL. Autopercepção de pessoas acometidas pela hanseníase sobre sua saúde bucal e necessidade de tratamento. Cienc Saude Colet 2013;18(3):817-26.

27. Mattos RA. Os sentidos da integralidade: algumas reflexões acerca de valores que merecem ser defendidos. Rio de Janeiro: Cepesc; 2006.

28. Fertonani HP, Pires DEP, Biff D, Scherer MDA. Modelo assistencial em saúde: conceitos e desafios para a atenção básica brasileira. Cienc Saude Colet 2015;20(6):1869-78.

29. Brasil. Ministério da Saúde. Agência Nacional de 
Vigilância Sanitária. Diretoria Colegiada. Portaria $\mathrm{n}^{\mathrm{O}} .389$ de 13 de março de 2014. Define os critérios para a organização da linha de cuidado da Pessoa com Doença Renal Crônica (DRC) e institui incentivo financeiro de custeio destinado ao cuidado ambulatorial pré-dialítico. 2014.

30. Blue C, Isringhausen K, Dils E. Raising oral health awareness among nephrology nurses. J Dent Hyg 2011;85(2):151-57.

31. Beber GC, Fontela PC, Herr GEG, Winkelmann ER. Qualidade de vida de pacientes transplantados renais após longo período do transplante. Saúde e Pesquisa 2017;10(1):163-70.

Recebido em: 14/10/2018

Aceito em: 28/12/2018 